\title{
Real Polynomial Root-finding by Means of Matrix and Polynomial Iterations*
}

\author{
Victor Y. Pan ${ }^{[1,2],[a]}$ and Liang Zhao ${ }^{[2],[b]}$ \\ [1] Department of Mathematics and Computer Science \\ Lehman College of the City University of New York \\ Bronx, NY 10468 USA \\ [2] Ph.D. Programs in Mathematics and Computer Science \\ The Graduate Center of the City University of New York \\ New York, NY 10036 USA \\ ${ }^{[a]}$ victor.pan@lehman.cuny.edu \\ http://comet.lehman.cuny.edu/vpan/ \\ ${ }^{[b]}$ lzhao1@gc.cuny.edu
}

\begin{abstract}
Univariate polynomial root-finding is a classical subject, still important for modern computing. Frequently one seeks just the real roots of a polynomial with real coefficients. They can be approximated at a low computational cost if the polynomial has no nonreal roots, but typically nonreal roots are much more numerous than the real ones. The subject of devising efficient real root-finders has been long and intensively studied. Nevertheless, we propose some novel ideas and techniques and obtain dramatic acceleration of the known numerical algorithms. In order to achieve our progress we exploit the correlation between the computations with matrices and polynomials, randomized matrix computations, and complex plane geometry, extend the techniques of the matrix sign iterations, and use the structure of the companion matrix of the input polynomial. The results of our extensive numerical tests with benchmark polynomials and random matrices are quite encouraging. In particular in these tests we have consistently computed accurate approximations of the real roots of benchmark polynomials of degree up to 1024 by using the IEEE standard double precision. Moreover the number of iterations required for convergence of our algorithms grew very slowly (if at all) as we increased the degree of the univariate input polynomials and the dimension of the input matrices from 64 to 1024 .
\end{abstract}

Keywords: Polynomials, Real roots, Matrices, Matrix sign iterations, Companion matrix, Frobenius algebra, Square root iterations, Root squaring

\section{Introduction}

Assume a univariate polynomial of degree $n$ with real coefficients,

$$
p(x)=\sum_{i=0}^{n} p_{i} x^{i}=p_{n} \prod_{j=1}^{n}\left(x-x_{j}\right), \quad p_{n} \neq 0,
$$

which has $r$ real roots $x_{1}, \ldots, x_{r}$ and $s=(n-r) / 2$ pairs of nonreal complex conjugate roots. In some applications, e.g., to algebraic and geometric optimization, one seeks only the $r$ real roots,

${ }^{*}$ This work appeared in Theoretical Computer Science, 2017, http://dx.doi.org/10.1016/j.tcs.2017.03.032. It has been supported by NSF Grants CCF 1116736 and CCF-1563942 and PSC CUNY Award 67699-00 45. Some of its results have been presented at CASC 2014. 
which typically make up just a small fraction of all roots 1 The design of efficient real root-finders is a well studied subject (see [19, Section 10.3.5], 47], [54, and the bibliography therein), but the most popular packages of subroutines for root-finding such as MPSolve 2000 [5], Eigensolve 2001 [21], and MPSolve 2012 [10] approximate the $r$ real roots about as fast and as slow as all the $n$ complex roots. It can be surprising, but we present some novel methods that accelerate the known numerical real root-finders by a factor of $n / r$, which is dramatic in various important applications.

The springboard for our real root-finders is the matrix sign iterations, which we apply to the companion matrix of an input polynomial. It is a well known technique for matrix computations 24, and we make it particularly efficient for real polynomial root-finding, although it has never been used for this purpose so far. By combining it with a number of known and novel techniques we ensure fast convergence of the iterations and their efficiency in numerical implementation with the IEEE standard double precision.

Our numerical tests confirm the efficiency of this approach. In particular, we closely approximate the real roots of various benchmark polynomials of degree up to 1024 by using double precision. Moreover the number of iterations required for convergence was typically quite small and grew very slowly (if it grew at all) as the polynomial degree increased from 64 to 1024 .

Some of our techniques should be of independent interest, e.g., our numerical stabilization in Section 3.3. our exploitation of matrix functions and randomized matrix computations in Algorithm 3.1. and the combination of our maps of the complex plane with rational transformations of the variable and the roots. Some of our algorithms (e.g., the ones of Section 3.4) combine operations with matrices and polynomials, demonstrating once again the value of synergistic combinations of this kind, which we have been advocating since [33] and [6].

Our goal in this paper is to present a novel approach to real root-finding for a polynomial and to demonstrate the promise of this approach by performing some preliminary tests. We hope that we have advanced toward our goals substantially, and there are promising directions for substantial improvement of the implementation of our algorithms. For example, Stage 3 of our Algorithm 3.1 is reduced to the inversion or orthogonalizaton of Toeplitz-like matrices, and the customary numerical algorithms, currently available for these operations, can be dramatically accelerated by means of the techniques of the papers [62] and 63.

We organize our paper as follows. In the next section we cover some background material. We present a variety of our real polynomial root-finders in Section 3. In Section 4 (the contribution of the second author) we present the results of our numerical tests. In the Appendix we cover some auxiliary results.

\section{Basic Definitions and Results}

Hereafter "flop" stands for "floating point arithmetic operation", assumed to be performed numerically, with bounded precision, e.g., the standard IEEE double precision.

\subsection{Some Basic Definitions for Matrix Computations}

- $\mathbb{C}^{m \times n}$ denotes the linear space of complex $m \times n$ matrices. $\mathbb{R}^{m \times n}$ is its subspace of $m \times n$ real matrices.

- $M^{T}=\left(m_{j i}\right)_{i, j=1}^{m, n}$ is the transpose of a matrix $M=\left(m_{i j}\right)_{i, j=1}^{m, n} \in \mathbb{C}^{m \times n} . M^{H}$ is its Hermitian transpose. $M^{H}=M^{T}$ for a real matrix $M$.

- $\|M\|=\|M\|_{2}$ denotes its spectral norm.

\footnotetext{
${ }^{1}$ Recall the following excerpt from [14]: "A celebrated result due to Erdös and Turán [20] says that, for a univariate polynomial over $\mathrm{C}$ whose middle coefficients are not too big with respect to its extremal coefficients, the arguments of its roots are approximately equidistributed. Combined with a recent result of Hughes and Nikeghbali [27, this shows that the roots of such a polynomial clustered near the unit circle." This result does not apply to some classes of polynomials of practical importance, but the study in [17] (also see the earlier papers [28] and [18]) shows that the expected number $r$ of the real roots of random real polynomials of various such classes stays in the range between orders of $\log (n)$ and $\sqrt{n}$; .
} 
- $I=I_{n}$ is the $n \times n$ identity matrix.

- $\operatorname{diag}\left(b_{j}\right)_{j=1}^{s}=\operatorname{diag}\left(b_{1}, \ldots, b_{s}\right)$ is the $s \times s$ diagonal matrix with the diagonal entries $b_{1}, \ldots, b_{s}$.

- $\mathcal{R}(M)$ is the range of a matrix $M$, that is, the linear space generated by its columns.

- A matrix of full column rank is a matrix basis of its range.

- A matrix $Q$ is unitary if $Q^{H} Q=I$ or $Q Q^{H}=I$, and such a matrix is called orthogonal if it is real.

- Suppose an $m \times n$ matrix $M$ has rank $n$ (and so $m \geq n$ ). Write $(Q, R)=(Q(M), R(M))$ to denote a unique pair of a unitary $m \times n$ matrix $Q$ and an upper triangular $n \times n$ matrix $R$ such that $M=Q R$ and all diagonal entries of the matrix $R$ are positive [22, Theorem 5.2.3].

- $M^{+}$is the unique Moore-Penrose pseudo inverse of $M$ [22, Section 5.5.2], equal to $M^{H}$ if and only if the matrix $M$ is unitary.

- An $m \times n$ matrix $M$ has an $n \times m$ left inverse matrix $X=M^{(I)}$ such that $X M=I_{n}$ if and only if it has full column rank $n$. In this case $M^{+}$is a left inverse. The left inverse is unique if and only if $M$ is a nonsingular matrix, in which case $m=n$ and $M^{(I)}=M^{-1}$.

- The $\epsilon$-rank of a matrix $M$ is the minimal rank of the matrices in its $\epsilon$-neighborhood. Numerical $\operatorname{rank} \operatorname{nrank}(M)$ is the $\epsilon$-rank where $\epsilon$ is small in context.

Definition 2.1. Eigenvalues, eigenvectors and eigenspaces.

- A scalar $x$ is an eigenvalue of a matrix $M$ associated with an eigenvector $\mathbf{v}$ if $M \mathbf{v}=x \mathbf{v}$.

- The eigenvectors associated with an eigenvalue $x$ or, more generally, with any set of the eigenvalues $\mathcal{X} \in \mathcal{X}(M)$ form the eigenspaces $\mathcal{S}(M, x)$ and $\mathcal{S}(M, \mathcal{X})$, respectively, associated with the eigenvalue $x$ and the set $\mathcal{X}$ of eigenvalues, respectively. A linear subspace $\mathcal{S}$ of $\mathbb{C}^{n \times n}$ is an eigenspace of a matrix $M$ if and only if $M \mathcal{S}=\{M \mathbf{v}: \mathbf{v} \in \mathcal{S}\} \subseteq \mathcal{S}$ (see [53, Definition 4.1.1]).

- An eigenvalue $x$ of a matrix $M$ is a root of the characteristic polynomial $\operatorname{det}(x I-M)$. The multiplicity of this root is the algebraic multiplicity of the eigenvalue $x$, denoted am $(x)$. The dimension $g m(x)=\operatorname{dim}(\mathcal{S}(M, x))$ is the geometric multiplicity of $x$, never exceeding am $(x)$. An eigenvalue $x$ is simple if $\operatorname{gm}(x)=1$.

\subsection{The Companion Matrix and the Frobenius Algebra}

Let $\mathbf{e}_{n}^{T}=(0,0, \ldots, 0,1)$ denote the $n$th coordinate vector and write $\mathbf{p}=\left(p_{i} / p_{n}\right)_{i=0}^{n-1}$,

$$
Z=C_{0}=\left(\begin{array}{ccccc}
0 & & & & 0 \\
1 & \ddots & & 0 \\
& \ddots & \ddots & & \vdots \\
& & \ddots & 0 & 0 \\
& & & 1 & 0
\end{array}\right) \text { and } C_{p}=\left(\begin{array}{ccccc}
0 & & & -p_{0} / p_{n} \\
1 & \ddots & & -p_{1} / p_{n} \\
& \ddots & \ddots & \vdots \\
& & \ddots & 0 & -p_{n-2} / p_{n} \\
& & & 1 & -p_{n-1} / p_{n}
\end{array}\right)=Z-\mathbf{p}^{T} \mathbf{e}_{n} .
$$

$Z$ is the down-shift matrix. $C_{p}$ is the companion matrix of the polynomial $p(x)$ of (1.1), which is the characteristic polynomial of this matrix. Hence real root-finding for the polynomial $p(x)$ turns into real eigen-solving for this matrix.

$Z \mathbf{v}=\left(v_{i-1}\right)_{i=1}^{n}$, for a vector $\mathbf{v}=\left(v_{i}\right)_{i=1}^{n}$ and for $v_{0}=0$.

Theorem 2.1. (The Cost of Computations in the Frobenius Matrix Algebra.) The companion matrix $C_{p} \in \mathbb{R}^{n \times n}$ of a polynomial $p(x)$ of (1.1) generates the Frobenius matrix algebra $\mathcal{A}_{p}$. One needs $O(n)$ flops for addition and $O(n \log (n))$ for multiplication and inversion in this algebra as well as for multiplication of a matrix in this algebra by a vector. These cost bounds hold both for exact computation with no errors and numerically stable approximate computations. 
Proof. The estimates for the exact computation can be readily deduced from the following expressions from [44] for a matrix $C(\mathbf{p}, \mathbf{c})$ in the algebra $\mathcal{A}_{p}$ defined by its first column $\mathbf{c}=\left(c_{i}\right)_{i=0}^{n-1}$,

$$
C(\mathbf{p}, \mathbf{c})=\sum_{i=0}^{n-1} c_{i} C_{p}^{i}=\sum_{i=0}^{n-1} c_{i} Z^{i}+\mathbf{p} \mathbf{u}^{T},
$$

for $\mathbf{p}$ of equation (2.1), $\mathbf{u}=\left(u_{i}\right)_{i=0}^{n-1}, u_{0}=0, u_{i}=\sum_{k=n-i}^{n-1} c_{k} \rho^{k-n+i}$, for $i=1, \ldots, n-1$ and $\rho=-p_{n-1} / p_{n}$, so that $\rho=0$ if $p_{n-1}=0$. (If $c_{0}=0$, then invert the matrix $C\left(\mathbf{q}, \mathbf{c}_{\mathbf{s}}\right)=C(\mathbf{p}, \mathbf{c})$ for $q(x)=p(x-s)$ and a random real or complex shift s.) The algorithm of [12], using the transition to the so called "Horner's basis", performs numerically stable multiplication in the algebra $\mathcal{A}_{p}$, 62. performs numerically stable inversion by using $O\left(n \log ^{2}(n)\right)$ flops, which is accelerated by a factor of $\log (n)$ in [43, Section 9.8].

\subsection{Decreasing the Size of an Eigenproblem}

An eigenvalue $x$ of a matrix $M$ as well as a set of eigenvalues $\mathcal{X}$ are dominant if they are absolutely larger than all the other eigenvalues. An eigenspace is called dominant if it is associated with a dominant eigenvalue or a dominant set of eigenvalues.

The set $\mathcal{X}(M)$ of all eigenvalues of a matrix $M$ is called its spectrum.

The Power Method [22, Section 7.3.1] computes the vector $M^{k} \mathbf{v}$, for a random vector $\mathbf{v}$ and a sufficiently large integer $k$. The 1-dimensional vector space $\left\{t M^{k} \mathbf{v}\right\}$, for $t \in \mathbb{C}$, is expected to approximate the eigenspace associated with an eigenvalue $x$ if it is dominant and simple. This would not work only if the vector $\mathbf{v}$ has an absolutely small component along the eigenvector associated with this eigenvalue $x$, but such an event is unlikely, for a random vector $\mathbf{v}$. One can choose $k=1$ if the domination of the eigenvalue $x$ in the spectrum of $M$ is strong. Let us extend the Power Method for $k=1$ to the approximation of a strongly dominant eigenspace of a dimension $r$.

Algorithm 2.1. Approximation of the dominant eigenspace.

INPUT: an $n \times n$ matrix $M$, the dimension $r$ of its dominant eigenspace $\mathcal{U}, 0<r<n$, and two tolerance bounds: a positive integer $K$ and a positive $\epsilon$.

OUTPUT: FAILURE (with a low probability) or a unitary matrix $U$ whose range approximates the eigenspace $\mathcal{U}$.

Computations:

1. Apply the randomized algorithm of [23], which at first generates a standard Gaussian random $n \times r_{+}$matrix $G$ for a proper integer $r_{+}>r$ and then computes the matrix $H=M G$ and the numerical rank $\operatorname{nrank}(H)$.

2. Unless $\operatorname{nrank}(H)=r$, re-apply the algorithm of [23] up to $K$ times until the equation $\operatorname{nrank}(H)=$ $r$ is observed. If it is never observed, output FAILURE (this occurs with a probability near 0).

3. If $\operatorname{nrank}(H)=r$, then compute the $Q R$ factorization $H=Q(H) R(H)$, output an $n \times r$ unitary matrix $U$ approximating the first $r$ columns of the matrix $Q(H)$, and stop. (The analysis in [23, Section 4], [45, Section 7.4], and [50, Theorem 4.3] shows that, with a probability close to 1 , the columns of the matrix $U$ closely approximate a unitary basis of the eigenspace $\mathcal{U}$ and that $\left\|M-U U^{H} M\right\| \leq \epsilon\|M\|$. The latter bound would certify correctness of the output.)

The arithmetic cost of performing the algorithm is $O\left(n^{2} r_{+}\right)$, but decreases to $O\left(n r_{+}\left(r_{+}+\log (n)\right)\right.$, for $M=C_{P}$, by virtue of Theorem [2.1. It increases by a factor of $\log (r)$ if the dimension $r$ of the eigenspace $\mathcal{U}$ is not available, but is computed by using binary search that begins with recursive doubling of the candidate integer values $1,2,4$, etc. The algorithm generates $n r_{+}$random parameters, but its modification using the structured (so called SRFT) multipliers $G$ involves only $n$ such parameters and only $O(n \log (n))$ flops for the computation of the product $M G$ (see 23, Section 11] and [45, Section 7.5]). Alternative application of the orthogonal iterations of [22, Section 7.3.2] requires order of $n^{2} r_{+}$flops. 
Remark 2.1. Actually the algorithm of [23] works even where the input includes an upper bound $r_{+}$on the dimension $r$ of the dominant eigenspace $\mathcal{U}$, rather than the dimension itself, and then the algorithm can compute this dimension $r$ within the above computational cost as by-product. (The integer $r=\operatorname{nrank}(H)$ can be obtained, e.g., from rank revealing $Q R$ factorization of the matrix $H$.

Now suppose that we have an eigenspace generated by $r$ eigenvalues of an $n \times n$ matrix. Then the following simple theorem (extending the recipe of the Rayleigh quotients) enables us to approximate these eigenvalues as the eigenvalues of an auxiliary $r \times r$ matrix.

Theorem 2.2. (Decreasing the Eigenproblem Size to the Dimension of an Eigenspace, cf. 660, Section 2.1].)

Suppose that $M \in \mathbb{C}^{n \times n}, U \in \mathbb{C}^{n \times r}$, and the matrix $U$ has full column rank $r \leq n$ and generates the space $\mathcal{U}=\mathcal{R}(U)$. Then

(i) $\mathcal{U}$ is an eigenspace of $M$ if and only if there exists a matrix $L \in \mathbb{C}^{k \times k}$ such that $M U=U L$ or equivalently if and only if $L=U^{(I)} M U$,

(ii) $\mathcal{X}(L) \subseteq \mathcal{X}(M)$,

(iii) $M U \mathbf{v}=x U \mathbf{v}$ if $L \mathbf{v}=x \mathbf{v}$,

(iv) the matrix $L$ is unique, that is, its choice is independent of the choice of a matrix $U$ and its left inverse $U^{(I)}$, and so $L=U^{H} M U$, for a unitary matrix $U$.

The algorithm and the theorem enable us to approximate the $r$ real eigenvalues of a matrix as the $r$ dominant eigenvalues of an auxiliary matrix. Theorems 2.2 and 2.3 (below) together suggest a direction to such a reduction, and we achieve it in Sections 3.1 and 3.3 .

\subsection{Matrix Functions and Eigenspaces}

Theorem 2.3. (The Eigenproblems for a Matrix and Its Function.)

Suppose that $M$ is a square matrix and that a rational function $f(x)$ is defined on its spectrum.

(i) Then $f(M) \mathbf{v}=f(x) \mathbf{v}$ if $M \mathbf{v}=x \mathbf{v}$.

(ii) Let $\mathcal{U}=\mathcal{U}_{\mu, f}$ denote the eigenspace of the matrix $f(M)$ associated with its eigenvalue $\mu$. Then this is an eigenspace of the matrix $M$ associated with all its eigenvalues $x$ such that $f(x)=\mu$.

(iii) The space $\mathcal{U}$ has dimension 1 and is associated with a single eigenvalue of $M$ if $\mu$ is a simple eigenvalue of $f(M)$.

Proof. We readily verify part (i), which implies parts (ii) and (iii).

Remark 2.2. The matrix $Z^{k}$, for $1 \leq k \leq n$, has the single eigenvalue 0 satisfying am $(0)=n$ and $\operatorname{gm}(0)=k$, and so $\operatorname{dim}\left(\mathcal{U}_{0, f}\right)=k$, for $M=Z, f(x)=x^{k}$, and $k=1, \ldots, n$.

Suppose that we have computed a matrix basis $U \in \mathbb{C}^{n \times r_{+}}$, for an eigenspace $\mathcal{U}$ of a matrix function $f(M)$ of an $n \times n$ matrix $M$. By virtue of Theorem 2.3, this is a matrix basis of an eigenspace of the matrix $M$. We can first compute a left inverse $U^{(I)}$ or the orthogonalization $Q=Q(U)$ and then approximate the eigenvalues of $M$ associated with this eigenspace as the eigenvalues of the $r_{+} \times r_{+}$matrix $L=U^{(I)} M U=Q^{H} M Q$ (cf. Theorem 2.2).

If $r=1$, then the matrix $U$ turns into an eigenvector $\mathbf{u}$, shared by the matrices $f(M)$ and $M$, while the matrix $L$ turns into the the Rayleigh Quotient $\frac{\mathbf{u}^{T} M \mathbf{u}}{\mathbf{u}^{T} \mathbf{u}}$ or the simple quotient $(M \mathbf{u})_{i} / u_{i}$, for any $i$ such that $u_{i} \neq 0$.

\subsection{Some Maps in the Frobenius Matrix Algebra}

Part (i) of Theorem 2.3 implies that, for a polynomial $p(x)$ of (1.1) and a rational function $f(x)$ defined on the set $\left\{x_{i}\right\}_{i=1}^{n}$ of its roots, the rational matrix function $f\left(C_{p}\right)$ has the spectrum $\mathcal{X}\left(f\left(C_{p}\right)\right)=$ $\left\{f\left(x_{i}\right)\right\}_{i=1}^{n}$. In particular, the maps

$$
C_{p} \rightarrow C_{p}^{-1}, C_{p} \rightarrow a C_{p}+b I, C_{p} \rightarrow C_{p}^{2}, C_{p} \rightarrow \frac{C_{p}+C_{p}^{-1}}{2}, \text { and } C_{p} \rightarrow \frac{C_{p}-C_{p}^{-1}}{2}
$$


induce the maps of the eigenvalues of the matrix $C_{p}$, and thus induce the maps of the roots of its characteristic polynomial $p(x)$ given by the equations

$$
y=1 / x, y=a x+b, y=x^{2}, y=0.5(x+1 / x), \text { and } y=0.5(x-1 / x),
$$

respectively. The latter two maps can be only applied if the matrix $C_{p}$ is nonsingular, so that $x \neq 0$, and similarly for the two dual maps below.

By using the reduction modulo $p(x)$, define the five dual maps

$$
\begin{gathered}
y=(1 / x) \bmod p(x), y=a x+b \quad \bmod p(x), y=x^{2} \quad \bmod p(x), \\
y=0.5(x+1 / x) \quad \bmod p(x), \text { and } y=0.5(x-1 / x) \quad \bmod p(x),
\end{gathered}
$$

where $y=y(x)$ denotes polynomials. Apply the two latter maps recursively, to define two iterations with polynomials modulo $p(x)$ as follows, $y_{0}=x, y_{h+1}=0.5\left(y_{h}+1 / y_{h}\right) \bmod p(x)$ and $y_{0}=$ $x, y_{h+1}=0.5\left(y_{h}-1 / y_{h}\right) \bmod p(x), h=0,1, \ldots$ More generally, define the iteration $y_{0}=x$, $y_{h+1}=a y_{h}+b / y_{h} \bmod p(x), h=0,1, \ldots$, for any pair of scalars $a$ and $b$, provided that $y_{h}=0$, for none $h$.

\section{Real Root-finders with Modified Matrix Sign Iterations. Variations and Extensions}

In this section we present some efficient numerical real root-finders based on modification of the matrix sign classical iterations applied to the companion matrix of the input polynomial.

\subsection{A Modified Matrix Sign Iterations}

Our first algorithm approximates the $r$ real roots of a polynomial $p(x)$ of (1.1) as the real eigenvalues of the companion matrix $C_{p}$. It applies the matrix iterations

$$
M_{0}=C_{p}, M_{h+1}=0.5\left(M_{h}-M_{h}^{-1}\right), \text { for } h=0,1, \ldots,
$$

which modify the matrix sign iterations $\widehat{M}_{h+1}=0.5\left(\widehat{M}_{h}+\widehat{M}_{h}^{-1}\right)$ (cf. 24]).

For every eigenvalue $x_{j}$ of the matrix $M_{0}=C_{p}$, define its trajectory made up of the eigenvalues of the matrices $M_{h}$, being its images in the maps $M_{0} \rightarrow M_{h}$, for $h=1,2,3, \ldots$ More generally iterations (3.2) below modifying the Möbius classical iterations $x^{(h+1)}=\frac{1}{2}\left(x^{(h)}+1 / x^{(h)}\right)$, for $h=$ $0,1, \ldots$, define a trajectory initiated at any complex point $x^{(0)}$.

Hereafter we write $\operatorname{sign}(z)=\operatorname{sign}(\Re(z))$, for a complex number $z$ (cf. [24, page 107]).

Theorem 3.1. (Convergence of the modified Möbius Iterations.) Fix a complex $x=x^{(0)}$ and define the modified Möbius iterations

$$
x^{(h+1)}=\frac{1}{2}\left(x^{(h)}-1 / x^{(h)}\right), \text { for } h=0,1, \ldots
$$

(i) The values $x^{(h)}$ are real, for all $h$, if $x^{(0)}$ is real.

(ii) $\left|x^{(h)}-\operatorname{sign}(x) \sqrt{-1}\right| \leq \frac{2 K^{2^{h}}}{1-K^{2^{h}}}$, for $K=\left|\frac{x-\operatorname{sign}(x)}{x+\operatorname{sign}(x)}\right|$ and $h=0,1, \ldots$

Proof. Part (i) is immediately verified. Part (ii) readily extends the similar estimate on [7, page 500].

Theorem 3.1 implies the following result.

Corollary 3.1. As $h \rightarrow \infty$, the trajectories of the $2 s$ nonreal eigenvalues of $M_{0}=C_{p}$ converge to $\pm \sqrt{-1}$ with the quadratic rate of convergence right from the start, whereas the trajectories of the $r$ real eigenvalues are real, for all $h$. 
Algorithm 3.1. Matrix sign iterations modified for real eigen-solving.

INPUT: two integers $n$ and $r, 0<r<n$, and the coefficients of a polynomial $p(x)$ of equation (1.1). OUtPUT: approximations to the real roots $x_{1}, \ldots, x_{r}$ of the polynomial $p(x)$ or FAILURE with a probability close to 0 .

Computations:

1. Write $M_{0}=C_{p}$ and recursively compute the matrices $M_{h+1}$ of (3.1), for $h=0,1, \ldots$ (cf. Corollary [3.1).

2. Fix a sufficiently large integer $k$ and compute the matrix $M=M_{k}^{2}+I_{n}$.

(By extending Corollary 3.1 observe that the map $M_{0}=C_{p} \rightarrow M$ sends all nonreal eigenvalues of $C_{p}$ into a small neighborhood of the origin 0 and sends all real eigenvalues of $C_{p}$ into the ray $\{x: x \geq 1\}$.)

3. Apply our randomized Algorithm 2.1 in order to approximate a unitary matrix $U$ whose columns form a basis for the eigenspace associated with the $r$ dominant eigenvalues of the matrix $M$.

(By virtue of Theorem 2.3, this is expected to be the eigenspace associated with the real eigenvalues of the matrix $C_{p}$, although with a probability close to 0 the algorithm can output FAILURE, in which case we stop the computations.)

4. Compute and output approximations to the $r$ eigenvalues of the $r \times r$ matrix $L=U^{H} C_{p} U$. (They approximate the $r$ real eigenvalues of the matrix $C_{p}$ by virtue of Theorem 2.2 and consequently approximate the $r$ real roots of the polynomial $p(x)$.)

Stages 1 and 2 involve $O(k n \log (n))$ flops by virtue of Theorem 2.1. Stage 3 adds $O\left(n^{2} r\right)$ flops and the cost $a_{r n}$ of generating $n \times r$ standard Gaussian random matrix. Add $O\left(r^{3}\right)$ flops performed at Stage 4 and arrive at the overall arithmetic cost bound $O\left(\left(k n \log (n)+n r^{2}\right)+a_{r n}\right.$.

Remark 3.1. (Counting Real Eigenvalues.) The binary search can produce the number of real eigenvalues as the numerical rank of the matrices $M_{k}^{2}+I$ when this rank stabilizes as $k$ increases. As the number of real roots increases, so does the size of the matrix L. This has consistently implied the decrease of the accuracy of the output approximations in our tests (see the test results in Section (4). One can refine these approximations by applying the inverse Power Method or Newton's iterations, but if the accuracy becomes too low, one must extend the precision of computing.

Remark 3.2. (Acceleration by Means of Scaling.) One can dramatically accelerate the initial convergence of Algorithm 3.1 by applying determinantal scaling (cf. [24]), that is, by replacing the matrix $M_{0}=C_{p}$ by the matrix $M_{0}=0.5\left(\nu C_{p}-\left(\nu C_{p}\right)^{-1}\right)$, for $\nu=1 /\left|\operatorname{det}\left(C_{p}\right)\right|^{1 / n}=\left|p_{n} / p_{0}\right|$.

Remark 3.3. Real and Nearly Real Roots.

In the presence of rounding errors Algorithm 3.1 and all our other algorithms approximate both $r$ real and $r_{+}-r$ nearly real eigenvalues of the matrix $M$, for some $r_{+} \geq r$. The $r$ real eigenvalues, however, are the roots of $p(x)$ and we can refine their approximations very fast (cf. Theorems B.1 and [48]), under some mild assumptions about the isolation of every such a root from the $n-1$ other roots. (One can partly relax these assumptions by extending the techniques of [48].) Then we can readily select the $r$ real eigenvalues among the $r_{+}$real and nearly real ones.

Generally, however, the distinction between real and nearly real roots is very slim in our numerical algorithms. As this was pointed out in [3], in the course of performing the iterations, the real eigenvalues can become nonreal, due to rounding errors, and then would converge to $\pm \sqrt{-1}$. In our extensive tests we have never observed such a phenomenon, apparently because in these tests the convergence to $\pm \sqrt{-1}$ was much slower for the nearly real eigenvalues than for the eigenvalues with reasonably large imaginary parts. 


\subsection{Inversion-free Variations of the Modified Matrix Sign Iterations and Hybrid Algorithms}

The overall arithmetic cost of the Modified Matrix Sign Iterations is dominated by the cost of $k$ matrix inversions, that is, $O\left(k n \log ^{2}(n)\right)$ flops (cf. Theorem 2.1). If all nonreal eigenvalues of the matrix $M_{0}$ lie in the two discs $D( \pm \sqrt{-1}, 1 / 2)=\{x:|x \pm \sqrt{-1}| \leq 1 / 2\}$, then we can avoid matrix inversions in the Modified Matrix Sign Iterations by replacing iterations (3.1) with any of the two iteration processes

$$
M_{h+1}=0.5\left(M_{h}^{3}+3 M_{h}\right)
$$

and

$$
M_{h+1}=-0.125\left(3 M_{h}^{5}+10 M_{h}^{3}+15 M_{h}\right),
$$

for $h=0,1, \ldots$. Right from the start both iterations send the nonreal roots toward the two points $\pm \sqrt{-1}$ with quadratic and cubic convergence rates, respectively. (In order to prove this, extend the proof of [7, Proposition 4.1].) Both iteration processes keep the real roots real and use $O(n \log (n))$ flops per iteration.

What if the nonreal roots do not lie in these discs? We can apply the following combination of iterations (3.1)-(3.4) and Corollary D.1 of Section D.

Algorithm 3.2. A Hybrid Algorithm.

InPut, OutPut as in Algorithm 3.1.

Computations: Perform the iterations of Algorithm 3.1 until a test shows that the $2 s$ nonreal eigenvalues of the input companion matrix are mapped into the discs $D( \pm \sqrt{-1}, 1 / 2)$. (For testing this condition, apply the algorithm that supports Corollary D.1. To keep the computational cost down, apply this test periodically, according to a fixed policy, based on heuristic rules or the statistics of the previous tests.) Then shift the computations to the inversion-free iterations (3.3) or (3.4) converging faster and using $O(n \log (n))$ flops per iteration.

Let us recall some alternative matrix iterations for real root-finding without inversions. Recall that $\operatorname{sign}(M)=M\left(M^{2}\right)^{-0.5}$ and apply the Newton-Schultz iterations for the approximation of the matrix square root [24, equation (6.3)],

$$
Y_{k+1}=0.5 Y_{k}\left(3 I-Z_{k} Y_{k}\right), \quad Y_{0}=M^{-2},
$$

and

$$
Z_{k+1}=0.5\left(3 I-Z_{k} Y_{k}\right) Z_{k}, \quad Z_{0}=I,
$$

for $k=0,1, \ldots$ The iterations keep real eigenvalues real and converge if $\left\|I-M^{-2}\right\|_{p}<1$, for $p=1,2$, or $\infty$. This assumption is easy to satisfy by means of scaling $M \rightarrow a M$, which keeps real eigenvalues real, for real $a$.

The similar coupling technique of $[3]$ is even simpler, because it is applied directly to the modified matrix sign iterations (3.1), preserving its quadratic convergence to $\pm \sqrt{-1}$ right from the start.

In our tests for numerical real root-finding, however, we could perform safely only a small number of these inversion-free iterations at the initial stage, and then the images of the real eigenvalues of the matrix $C_{p}$ grew very large and the condition numbers of the computed matrices blew up.

\subsection{Numerical Stabilization of the Modified Matrix Sign Iterations}

The images of nonreal eigenvalues of the matrix $C_{p}$ converge to $\pm \sqrt{-1}$ in the iterations of Stage 1 of Algorithm 3.1] but if the images of some real eigenvalues of $C_{p}$ come close to 0 , then at the next step we would have to invert an ill-conditioned matrix $M_{h}$ unless we are applying an inversion-free variant of the iterations of the previous subsection.

We can try to avoid this problem by shifting the matrix (and its eigenvalues), that is, by adding to the current matrix $M_{h}$ the matrix $s I$, for a reasonably small positive scalar $s$ or $-s$. We can select this scalar by applying heuristic methods or randomization. In our tests this policy has preserved convergence quite well, but we have no formal support for this observation. The following 
stabilization of Algorithm 3.1 involves nonreal values even when the matrix $C_{p}$ was real, but has both formal and empirical support.

Algorithm 3.3. Numerical stabilization of the modified matrix sign iterations.

InPut, Output and Stages 3 and 4 of Computations are as in Algorithm 3.1, except that the input includes a small positive scalar $\alpha$ such that no eigenvalues of the matrix $C_{p}$ have imaginary parts close to $\pm \alpha \sqrt{-1}$ (see Remark 3.4 below), the set of $r$ real roots $x_{1}, \ldots, x_{r}$ of the polynomial $p(x)$ is replaced by the set of its $r_{+}$roots having the imaginary parts in the range $[-\alpha, \alpha]$, and the integer $r$ is replaced by the integer $r_{+}$throughout.

Computations:

1. Apply Stage 1 of Algorithm 3.1 to the two matrices $M_{0, \pm}=\alpha \sqrt{-1} I \pm C_{p}$, thus producing two sequences of the matrices $M_{h,+}$ and $M_{h,-}$, for $h=0,1, \ldots$.

2. Fix a sufficiently large integer $k$ and compute the matrix $M=M_{k,+}+M_{k,-}$.

Because of the assumed choice of $\alpha$, the matrices $\alpha \sqrt{-1} I \pm C_{p}$ have no real eigenvalues, and so the images of all their eigenvalues, that is, the eigenvalues of the matrices $M_{k,+}$ and $M_{k,-}$, converge to $\pm \sqrt{-1}$ as $k \rightarrow \infty$. Moreover, one can verify that the eigenvalues of the matrix $M_{k,+}+M_{k,-}$ converge to 0 unless they are the images of the $r_{+}$eigenvalues of the matrix $C_{p}$ having the imaginary parts in the range $[-\alpha, \alpha]$. The latter eigenvalues of the matrix $M_{k,+}+M_{k,-}$ converge to $2 \sqrt{-1}$. This shows correctness and numerical stability of Algorithm 3.3 .

The algorithm approximates the $r_{+}$roots of $p(x)$ by using $O\left(k n \log (n)+n r_{+}^{2}\right)+a_{r_{+} n}$ flops, versus $O\left(k n \log (n)+n r^{2}\right)+a_{r n}$ involved in Algorithm 3.1

Remark 3.4. We can test the proximity of the roots to a line in two stages: by at first moving the line into the unit circle $\{x:|x|=1\}$ (cf. Theorem A.3) and then applying algorithms that supports Theorem D.2 or Corollary D.1.

\subsection{Square Root Iterations (a Modified Modular Version)}

Next we describe a dual polynomial version of Algorithm 3.1. It extends the square root iterations $y_{h+1}=\frac{1}{2}\left(y_{h}+1 / y_{h}\right), h=0,1, \ldots$, and at Stage 2 involves the computation of the polynomial $\operatorname{agcd}\left(p, t_{k}\right)$, which denotes an approximate greatest common divisor of the input polynomial $p=p(x)$ and an auxiliary polynomial $t_{k}=t_{k}(x)$. We refer the reader to [38, [29], 4], 61], [1], and [55] for the definitions of this concept and the algorithms for its computation.

Compared to Algorithm [3.1, we replace all rational functions in the matrix $C_{p}$ by the same rational functions in the variable $x$ and reduce them modulo the input polynomial $p(x)$. The reduction does not affect the values of the functions at the roots of $p(x)$, and it follows that these values are precisely the eigenvalues of the rational matrix functions computed in Algorithm 3.1 .

Algorithm 3.4. Square root modular iterations modified for real root-finding.

INPUT: two integers $n$ and $r, 0<r<n$, and the coefficients of a polynomial $p(x)$ of equation (1.1).

OUtPut: approximations to the real roots $x_{1}, \ldots, x_{r}$ of the polynomial $p(x)$.

Computations:

1. (Cf. (3.1).) Write $y_{0}=x$ and compute the polynomials

$$
y_{h+1}=\frac{1}{2}\left(y_{h}-1 / y_{h}\right) \bmod p(x), h=0,1, \ldots
$$

2. Periodically, for some selected integers $k$, compute the polynomials $t_{k}=y_{k}^{2}+1 \bmod p(x)$.

3. Write $g_{k}(x)=\operatorname{agcd}\left(p, t_{k}\right)$ and compute $d_{k}=\operatorname{deg}\left(g_{k}(x)\right)$. If $d_{k}=n-r=2 s$, compute the polynomial $v_{k} \approx p(x) / g_{k}(x)$ of degree $r$. Otherwise continue the iterations of Stage 1 . 
4. Apply one of the algorithms of [1], [8], and [15] (cf. Theorem [C.1) to approximate the roots $y_{1}, \ldots, y_{r}$ of the polynomial $v_{k}$. Output these approximations.

Our comments preceding this algorithm show that the values of the polynomials $t_{k}(x)$ at the roots of $p(x)$ are equal to the images of the eigenvalues of the matrix $C_{p}$ in Algorithm 3.1. Hence the values of the polynomials $t_{k}(x)$ at the nonreal roots of $p(x)$ converge to 0 as $k \rightarrow \infty$, whereas their values at the real roots of $p(x)$ stay far from 0 . Therefore, for sufficiently large integers $k$, $\operatorname{agcd}\left(p, t_{k}\right)$ turns into the polynomial $\prod_{j=r+1}^{n}\left(x-x_{j}\right)$. This implies correctness of the algorithm.

Its asymptotic computational cost is $O\left(k n \log ^{2}(n)\right)$ plus the cost of computing $\operatorname{agcd}\left(p, t_{k}\right)$ and choosing the integer $k$ (see our next remark).

Remark 3.5. The latter algorithm reduces real root-finding essentially to the computation of agcd $\left(p, t_{k}\right)$. One can apply quite efficient heuristic algorithms for this computation (cf. [38], [29], [4], [61], [11], and [55]), but no good formal estimates are available for their complexity. One can, however, note that $p(x) u_{k}(x) \approx t_{k}(x) v_{k}(x)$, and so, assuming that $v_{k}(x)$ is a monic polynomial (otherwise we can scale it), can obtain its other coefficients (as well as the coefficients of the polynomial $u_{k}(x)$ ) from the least-squares solution to the associated Sylvester linear system of equations. Its well known superfast divide and conquer solution involves order of $n \log ^{2}(n)$ arithmetic operations (cf. [36. Chapter 5]), but the recent numerically stable algorithm of [62] accelerated by a factor of $\log (n)$ in [43, Section $9.8]$ involves only $O(n \log (n))$ flops.

\section{Numerical Tests}

Extensive numerical tests of the algorithms of this paper, performed in the Graduate Center of the City University of New York. They are the contribution of the second author (at some points he was assisted by Ivan Retamoso). The tests recorded the number of iterations and the error of the approximation of the real roots of benchmark polynomials to which we applied these algorithms. We have recorded similar data also for the approximation of real eigenvalues of some random matrices $M$ by means of applying Algorithms 3.1 and 3.3. In the latter case the convergence of these algorithms and the number of their iterations depended mostly on the characteristic polynomials of $M$, even though the estimates for the arithmetic cost of performing each iteration generally grew compared to the special case where $M=C_{p}$.

In some cases we stopped the iterations already when they produced crude approximation to the roots. This is because, instead of continuing the iterations, we can apply the algorithms of 48 followed by Newton's or Ehrlich-Aberth's iterations (cf. Section B), which refine very fast these crude approximations.

Finally we note that the test results in the present section are quite encouraging (in spite of our caveat in Remark 3.1), e.g., the numbers of iterations required for convergence of our algorithms have grown very slowly (if at all) when we increased the degree of the input polynomials and dimension of the input matrices from 64 to 1024. We performed all tests with the IEEE standard double precision.

The implementation is available upon request.

\subsection{Tests for the Modified Matrix Sign Iterations (Algorithm 3.1)}

In the first series of the tests, Algorithm 3.1 has been applied to one of the Mignotte benchmark polynomials, namely $p(x)=x^{n}+(100 x-1)^{3}$. It is known that this polynomial has three illconditioned roots clustered about 0.01 and has $n-3$ well-conditioned roots. In the tests, Algorithm 3.1 has output the roots within the error less than $10^{-6}$ by using 9 iterations, for $n=32$ and $n=64$ and by using 11 iterations, for $n=128$ and $n=256$.

In the second series of the tests, polynomials $p(x)$ of degree $n=50,100,150,200$, and 250 have been generated as the products $p(x)=p_{1}(x) p_{2}(x)$, for the $r$ th degree Chebyshev polynomial $p_{1}(x)$ (having $r$ real roots), $r=8,12,16$, and $p_{2}(x)=\sum_{i=0}^{n-r} a_{i} x^{i}, a_{j}$ being i.i.d. standard Gaussian random variables, for $j=0, \ldots, n-r$. Algorithm 3.1 (performed with double precision) was applied to 100 such polynomials $p(x)$, for each pair of $n$ and $r$. Table 4.1 displays the output data, namely, 
the average values and the standard deviation of the numbers of iterations and of the maximum difference between the output values of the roots and their values produced by MATLAB root-finding function "roots()".

In the third series of the tests, Algorithm 3.1 approximated the real eigenvalues $x_{1}, \ldots, x_{r}$ of a random complex symmetric matrix $A=U^{T} \Sigma U$, for $\Sigma=\operatorname{diag}\left(x_{1}, \ldots, x_{r}, y_{1}, \ldots, y_{n-r}\right)$, $r$ i.i.d. real standard Gaussian random variables $x_{1}, \ldots, x_{r}, n-r$ i.i.d. complex (non-real) standard Gaussian random variables $y_{1}, \ldots, y_{n-r}$, and a $n \times n$ standard Gaussian random orthogonal matrix $U$. Table 4.2 displays the mean and standard deviation of the number of iterations and the error bounds in these tests, for $n=50,100,150,200,250$ and $r=8,12,16$.

In order to estimate the number of iterations required in our algorithms, we periodically estimated the numerical rank of the associated matrix in every $k$ successive iterations, for $k=5$ in most of our experiments.

Table 4.1: Number of Iterations and Error Bounds for Algorithm 3.1 on Random Polynomials

\begin{tabular}{|c|c|c|c|c|c|}
\hline $\mathbf{n}$ & $\mathbf{r}$ & Iteration-mean & Iteration-std & Error-mean & Error-std \\
\hline 50 & 8 & 7.44 & 1.12 & $4.18 \times 10^{-6}$ & $1.11 \times 10^{-5}$ \\
\hline 100 & 8 & 8.76 & 1.30 & $5.90 \times 10^{-6}$ & $1.47 \times 10^{-5}$ \\
\hline 150 & 8 & 9.12 & 0.88 & $2.61 \times 10^{-5}$ & $1.03 \times 10^{-4}$ \\
\hline 200 & 8 & 9.64 & 0.86 & $1.48 \times 10^{-6}$ & $5.93 \times 10^{-6}$ \\
\hline 250 & 8 & 9.96 & 0.73 & $1.09 \times 10^{-7}$ & $5.23 \times 10^{-5}$ \\
\hline 50 & 12 & 7.16 & 0.85 & $3.45 \times 10^{-4}$ & $9.20 \times 10^{-4}$ \\
\hline 100 & 12 & 8.64 & 1.15 & $1.34 \times 10^{-5}$ & $2.67 \times 10^{-5}$ \\
\hline 150 & 12 & 9.12 & 2.39 & $3.38 \times 10^{-4}$ & $1.08 \times 10^{-3}$ \\
\hline 200 & 12 & 9.76 & 2.52 & $6.89 \times 10^{-6}$ & $1.75 \times 10^{-5}$ \\
\hline 250 & 12 & 10.04 & 1.17 & $1.89 \times 10^{-5}$ & $4.04 \times 10^{-5}$ \\
\hline 50 & 16 & 7.28 & 5.06 & $3.67 \times 10^{-3}$ & $7.62 \times 10^{-3}$ \\
\hline 100 & 16 & 10.20 & 5.82 & $1.44 \times 10^{-3}$ & $4.51 \times 10^{-3}$ \\
\hline 150 & 16 & 15.24 & 6.33 & $1.25 \times 10^{-3}$ & $4.90 \times 10^{-3}$ \\
\hline 200 & 16 & 13.36 & 5.38 & $1.07 \times 10^{-3}$ & $4.72 \times 10^{-3}$ \\
\hline 250 & 16 & 13.46 & 6.23 & $1.16 \times 10^{-4}$ & $2.45 \times 10^{-4}$ \\
\hline
\end{tabular}


Table 4.2: Number of Iterations and Error Bounds for Algorithm 3.1 on Random Matrices

\begin{tabular}{|c|c|c|c|c|c|}
\hline $\mathbf{n}$ & $\mathbf{r}$ & Iteration-mean & Iteration-std & Error-mean & Error-std \\
\hline 50 & 8 & 10.02 & 1.83 & $5.51 \times 10^{-11}$ & $1.65 \times 10^{-10}$ \\
\hline 100 & 8 & 10.81 & 2.04 & $1.71 \times 10^{-12}$ & $5.24 \times 10^{-12}$ \\
\hline 150 & 8 & 14.02 & 2.45 & $1.31 \times 10^{-13}$ & $3.96 \times 10^{-13}$ \\
\hline 200 & 8 & 12.07 & 0.94 & $2.12 \times 10^{-11}$ & $6.70 \times 10^{-11}$ \\
\hline 250 & 8 & 13.59 & 1.27 & $2.75 \times 10^{-10}$ & $8.14 \times 10^{-10}$ \\
\hline 50 & 12 & 10.46 & 1.26 & $1.02 \times 10^{-12}$ & $2.61 \times 10^{-12}$ \\
\hline 100 & 12 & 10.60 & 1.51 & $1.79 \times 10^{-10}$ & $3.66 \times 10^{-10}$ \\
\hline 150 & 12 & 11.25 & 1.32 & $5.69 \times 10^{-8}$ & $1.80 \times 10^{-7}$ \\
\hline 200 & 12 & 12.36 & 1.89 & $7.91 \times 10^{-10}$ & $2.50 \times 10^{-9}$ \\
\hline 250 & 12 & 11.72 & 1.49 & $2.53 \times 10^{-12}$ & $3.84 \times 10^{-12}$ \\
\hline 50 & 16 & 10.10 & 1.45 & $1.86 \times 10^{-9}$ & $5.77 \times 10^{-9}$ \\
\hline 100 & 16 & 11.39 & 1.70 & $1.37 \times 10^{-10}$ & $2.39 \times 10^{-10}$ \\
\hline 150 & 16 & 11.62 & 1.78 & $1.49 \times 10^{-11}$ & $4.580 \times 10^{-11}$ \\
\hline 200 & 16 & 11.88 & 1.32 & $1.04 \times 10^{-12}$ & $2.09 \times 10^{-12}$ \\
\hline 250 & 16 & 12.54 & 1.51 & $3.41 \times 10^{-11}$ & $1.08 \times 10^{-10}$ \\
\hline
\end{tabular}




\subsection{Tests for the Stabilized Matrix Sign Iterations (Algorithm 3.3) Applied to Polynomials}

We tested Algorithm 3.3 on various modified benchmark polynomials from the website of MPSolve (http://numpi.dm.unipi.it/mpsolve-2.2/). With the exception of the polynomials of Type IV below, we tested benchmark polynomials that had only trivial real roots 0 and \pm 1 , and we multiplied them by Chebyshev polynomials of degree $r$, for $r=8,12$, and 16, which have only real roots.

Having generated such a polynomial $p=p(x)$ and its companion matrix $C_{p}$, we computed the condition numbers of the matrices $N_{k}=C_{p}+2^{7+k} I_{n}$ with $k=1,2, \ldots$ and selected an integer $k$ such that $\kappa\left(N_{k}\right)<10^{5}$. Clearly, this is ensured for sufficiently large integers $k$ defining diagonally dominant matrices $N_{k}$, but in our tests $k$ was less than five in most cases.

Having fixed $k$ and $N_{k}$ and following the description of Algorithm 3.3, we computed at first the matrices $Y_{1}=\alpha I_{n}+N_{k}$ and $Y_{2}=\alpha I_{n}-N_{k}$, for $\alpha=0.0001 \sqrt{-1}$, and then successively the matrices $Y_{i+1, j}=\frac{1}{2}\left(Y_{i, j}-Y_{i, j}^{-1}\right)$ with $Y_{0, j}=Y_{j}$, for $j=1,2$ (cf. Algorithms 3.1 and 3.3).

We have observed that with our real shifts by $2^{7+k} I_{n}$ at the initial stage, non-real eigenvalues of $Y_{1}$ and $Y_{2}$ were never close to $\pm \sqrt{-1}$ at the first $7+k$ iterations. So we began checking convergence only when we have performed these initial iterations, and since that moment we checked convergence in every five iterations. As soon as we observed that $\operatorname{nrank}\left(Y_{i}^{\prime}\right)=r$, for $Y_{i}^{\prime}=Y_{i, 1}+Y_{i, 2}$ and for $r$ denoting the number of distinct real roots of $p(x), r=8,12,16$, we stopped the iterations and moved to the final stage of the algorithm, that is, approximated the real eigenvalues of matrix $C_{p}$, equal to the real roots of the polynomial $p(x)$.

We have run numerical tests on polynomials of five types having degree $n=64,128,256,512,1024$, and we compared our results with the outputs of MATLAB function " $\operatorname{roots}()$ ". We defined polynomial $p(x)$ of Types I-III and V as the products $p(x)=p_{1}(x) p_{2}(x)$ where $p_{1}(x)$ is the $r$-th degree Chebyshev polynomial and $p_{2}(x)$ are the following polynomials:

I. $p_{2}(x)=x^{n-r}-1$,

II. $p_{2}(x)=1+2 x+3 x^{2}+\cdots+(n-r+1) x^{n-r}$,

III. $p_{2}(x)=(x+1)(x+a)\left(x+a^{2}\right) \cdots\left(x+a^{n-r-1}\right)$, with $a=\frac{i}{100}$, and

V. $p_{2}(x)=\sum_{k=0}^{n} a_{k} x^{k}$, with $a_{0}, \ldots, a_{n}$ being i.i.d. standard random variables.

We also tested the following polynomials of Type IV,

IV. $p(x)=x^{n-r}-(a x-1)^{3}$, where $a=60,80,100$.

Tables 4.3 4.6 display the number of iterations and the maximum error bounds, for the polynomials of Types I-IV (cf. our Remark 3.1). Table 4.7 shows the average error bounds and the average numbers of iterations in 50 tests with the polynomials of Type V. 
Table 4.3: Number of Iterations and Error Bounds for Algorithm 3.3 on Type I Polynomials

\begin{tabular}{|c|c|c|c|}
\hline $\mathbf{n}$ & $\mathbf{r}$ & Iterations & Errors \\
\hline 64 & 8 & 10 & $1.03 E-10$ \\
\hline 64 & 12 & 23 & $1.32 E-08$ \\
\hline 64 & 16 & 23 & $3.97 E-06$ \\
\hline 128 & 8 & 10 & $1.60 E-10$ \\
\hline 128 & 12 & 23 & $4.91 E-04$ \\
\hline 128 & 16 & 23 & $2.22 E-03$ \\
\hline 256 & 8 & 10 & $6.18 E-06$ \\
\hline 256 & 12 & 28 & $1.75 E-09$ \\
\hline 256 & 16 & 28 & $3.54 E-06$ \\
\hline 512 & 8 & 15 & $8.05 E-13$ \\
\hline 512 & 12 & 28 & $1.71 E-08$ \\
\hline 512 & 16 & 28 & $2.78 E-05$ \\
\hline 1024 & 8 & 15 & $2.33 E-12$ \\
\hline 1024 & 12 & 28 & $1.27 E-09$ \\
\hline 1024 & 16 & 28 & $2.19 E-05$ \\
\hline
\end{tabular}

Table 4.4: Number of Iterations and Error Bounds for Algorithm 3.3 on Type II Polynomials

\begin{tabular}{|c|c|c|c|}
\hline $\mathbf{n}$ & $\mathbf{r}$ & Iterations & Errors \\
\hline 64 & 8 & 10 & $1.53 E-11$ \\
\hline 64 & 12 & 23 & $1.30 E-07$ \\
\hline 64 & 16 & 23 & $1.40 E-05$ \\
\hline 128 & 8 & 28 & $9.42 E-11$ \\
\hline 128 & 12 & 10 & $7.51 E-08$ \\
\hline 128 & 16 & 28 & $2.27 E-04$ \\
\hline 256 & 8 & 28 & $1.92 E-11$ \\
\hline 256 & 12 & 28 & $2.21 E-07$ \\
\hline 256 & 16 & 28 & $1.69 E-03$ \\
\hline 512 & 8 & 28 & $3.68 E-12$ \\
\hline 512 & 12 & 28 & $2.17 E-06$ \\
\hline 512 & 16 & 33 & $1.53 E-02$ \\
\hline 1024 & 8 & 28 & $2.96 E-11$ \\
\hline 1024 & 12 & 33 & $5.00 E-07$ \\
\hline 1024 & 16 & 33 & $3.58 E-03$ \\
\hline
\end{tabular}


Table 4.5: Number of Iterations and Error Bounds for Algorithm 3.3 on Type III Polynomials

\begin{tabular}{|c|c|c|c|}
\hline $\mathbf{n}$ & $\mathbf{r}$ & Iterations & Errors \\
\hline 64 & 8 & 28 & $4.63 E-11$ \\
\hline 64 & 12 & 23 & $1.69 E-07$ \\
\hline 64 & 16 & 28 & $7.36 E-06$ \\
\hline 128 & 8 & 28 & $3.83 E-12$ \\
\hline 128 & 12 & 23 & $1.45 E-08$ \\
\hline 128 & 16 & 28 & $1.68 E-05$ \\
\hline 256 & 8 & 28 & $1.58 E-12$ \\
\hline 256 & 12 & 23 & $1.02 E-04$ \\
\hline 256 & 16 & 28 & $6.50 E-04$ \\
\hline 512 & 8 & 28 & $7.69 E-13$ \\
\hline 512 & 12 & 23 & $5.00 E-09$ \\
\hline 512 & 16 & 28 & $8.60 E-06$ \\
\hline 1024 & 8 & 28 & $9.90 E-14$ \\
\hline 1024 & 12 & 23 & $1.45 E-09$ \\
\hline 1024 & 16 & 28 & $2.64 E-05$ \\
\hline
\end{tabular}

Table 4.6: Number of Iterations and Error Bounds Algorithm 3.3 on Type IV Polynomials

\begin{tabular}{|c|c|c|c|}
\hline $\mathbf{n}$ & $\mathbf{a}$ & Iterations & Errors \\
\hline 64 & 60 & 41 & $2.43 E-04$ \\
\hline 64 & 80 & 42 & $7.98 E-04$ \\
\hline 64 & 100 & 43 & $1.72 E-05$ \\
\hline 128 & 60 & 41 & $1.12 E-03$ \\
\hline 128 & 80 & 42 & $4.43 E-04$ \\
\hline 128 & 100 & 43 & $1.31 E-04$ \\
\hline 256 & 60 & 41 & $2.10 E-04$ \\
\hline 256 & 80 & 42 & $1.91 E-04$ \\
\hline 256 & 100 & 43 & $1.34 E-04$ \\
\hline 512 & 60 & 41 & $3.37 E-04$ \\
\hline 512 & 80 & 42 & $1.80 E-04$ \\
\hline 512 & 100 & 43 & $8.33 E-05$ \\
\hline 1024 & 60 & 36 & $1.10 E-01$ \\
\hline 1024 & 80 & 42 & $1.16 E-04$ \\
\hline 1024 & 100 & 43 & $1.76 E-04$ \\
\hline
\end{tabular}


Table 4.7: Number of Iterations and Error Bounds for Algorithm 3.3 on Type V Polynomials

\begin{tabular}{|c|c|c|c|}
\hline $\mathbf{n}$ & $\mathbf{r}$ & Iterations & Errors \\
\hline 128 & 8 & 22.3 & $5.33 E-06$ \\
\hline 128 & 12 & 24.6 & $4.85 E-05$ \\
\hline 128 & 16 & 24.94 & $3.59 E-03$ \\
\hline 256 & 8 & 26.02 & $1.11 E-06$ \\
\hline 256 & 12 & 27.01 & $2.37 E-05$ \\
\hline 256 & 16 & 30.18 & $1.80 E-03$ \\
\hline 512 & 8 & 27.54 & $2.73 E-08$ \\
\hline 512 & 12 & 28.00 & $2.27 E-06$ \\
\hline 512 & 16 & 38.18 & $2.39 E-03$ \\
\hline
\end{tabular}




\subsection{Tests for the Stabilized Matrix Sign Iterations (Algorithm 3.3) on Gaussian Random Matrices}

We tested Algorithm 3.3 on randomly generated matrices of two types:

Type I: Gaussian random tridiagonal matrices of dimension $n=64,128,256,512,1024$. We generated each entry in the tridiagonal part independently by using standard Gaussian distribution and set the other entries to 0 . Our tables show the error bounds equal to the maximal difference of the outputs of our algorithm and MATLAB function "eig()". We generated 100 matrices, for each $n$, and recorded the mean and standard deviation of the error bounds and of the numbers of iterations.

Type II: Random matrices $A$ with a fixed number of real eigenvalues. At first we generated a diagonal matrix $\Sigma$ with $r$ diagonal entries under the standard real Gaussian distribution and $n-r$ diagonal entries under the standard complex Gaussian distribution, for $n=64,128,256,512,1024$ and $r=8,12,16$. Then we generated a standard Gaussian random orthogonal matrix $Q$. Finally we computed the matrices $A=Q^{T} \Sigma Q$. We generated 100 such matrices $A$, for each pair of $n$ and $r$, and recorded the mean and standard deviation of the error bounds and of the numbers of iterations.

The following two tables summarize the performance data, showing a low number of iterations required for ensuring the approximation of the eigenvalues with a reasonable precision.

Table 4.8: Number of Iterations and Error Bounds for Root-finding Algorithm 3.3 on Type I matrices

\begin{tabular}{|c|c|c|c|c|}
\hline $\mathbf{n}$ & Iteration-mean & Iteration-std & Error-mean & Error-std \\
\hline 64 & 10.70 & 2.36 & $1.78 E-06$ & $1.14 E-05$ \\
\hline 128 & 12.16 & 3.34 & $5.68 E-07$ & $4.49 E-06$ \\
\hline 256 & 12,97 & 3.97 & $3.26 E-06$ & $1.35 E-05$ \\
\hline 512 & 15.46 & 9.82 & $8.80 E-04$ & $8.44 E-03$ \\
\hline 1024 & 16.52 & 10.26 & $2.43 E-03$ & $2.25 E-02$ \\
\hline
\end{tabular}

Table 4.9: Number of Iterations and Error Bounds for Algorithm 3.3 on Type II matrices

\begin{tabular}{|c|c|c|c|c|c|}
\hline $\mathbf{n}$ & $\mathbf{r}$ & Iteration-mean & Iteration-std & Error-mean & Error-std \\
\hline 64 & 8 & 11.65 & 2.47 & $3.69 E-08$ & $2.29 E-07$ \\
\hline 64 & 12 & 11.75 & 2.50 & $3.98 E-10$ & $2.71 E-09$ \\
\hline 64 & 16 & 11.60 & 2.45 & $4.10 E-09$ & $3.88 E-08$ \\
\hline 128 & 8 & 13.75 & 2.79 & $1.17 E-08$ & $7.56 E-08$ \\
\hline 128 & 12 & 13.70 & 2.90 & $4.41 E-09$ & $2.73 E-08$ \\
\hline 128 & 16 & 13.65 & 2.55 & $1.23 E-07$ & $1.34 E-06$ \\
\hline 256 & 8 & 14.55 & 3.26 & $5.59 E-09$ & $5.58 E-08$ \\
\hline 256 & 12 & 14.15 & 3.70 & $1.38 E-07$ & $1.38 E-06$ \\
\hline 256 & 16 & 14.70 & 2.54 & $3.06 E-11$ & $1.93 E-10$ \\
\hline 512 & 8 & 13.65 & 5.59 & $5.08 E-10$ & $4.88 E-09$ \\
\hline 512 & 12 & 15.65 & 9.47 & $7.46 E-04$ & $7.46 E-03$ \\
\hline 512 & 16 & 16.55 & 10.26 & $2.78 E-03$ & $5.47 E-03$ \\
\hline 1024 & 8 & 18.20 & 15.35 & $2.33 E-10$ & $1.22 E-09$ \\
\hline 1024 & 12 & 20.85 & 17.60 & $1.27 E-07$ & $3.36 E-07$ \\
\hline 1024 & 16 & 24.35 & 19.56 & $2.19 E-03$ & $4.33 E-03$ \\
\hline
\end{tabular}




\subsection{Tests for the Hybrid Matrix Algorithm (Algorithm 3.2) on Bench- mark Polynomials}

We performed numerical tests of a hybrid algorithm. We began with Algorithm 3.1 and after sufficiently many iterations continued with its variation avoiding matrix inversion.

Namely, we first applied a real shift $\beta I$ to the companion matrix $C_{p}$, such that the matrix $M=C_{p}+\beta I$ had condition number less than $10^{5}$. Based on our previous tests, we expected that, for such inputs, at least $T=\log _{2} \beta$ iterations $M_{i+1}=\frac{1}{2}\left(M_{i}-M_{i}^{-1}\right)$ would be required in order to move the complex nonreal eigenvalues close enough to $\pm \sqrt{-1}$. After the first $T$ iterations, we periodically (in every 5 iterations) applied two iterations $M_{i+1}=\frac{1}{2}\left(M_{i}^{3}+3 M_{i}\right)$, which converged with cubic rate provided that all complex eigenvalues have distance less than $\frac{1}{2}$ from $\sqrt{-1}$ or $-\sqrt{-1}$. Before switching to the iterations of the second type, we performed the following transformation in order to avoid problems of numerical stability:

Step 1: Compute $P=\frac{0.5 M+\sqrt{-1} I}{0.5 M+\sqrt{-1} I}$, which maps the real line into the unit circle.

Step 2: Compute $Y=\frac{2 \sqrt{-1}}{3}\left(P-P^{-1}\right)$, mapping the unit circle onto the interval $[-2 / 3,2 / 3]$.

Note that these two maps together keep the values $\pm \sqrt{-1}$ unmoved.

We tested polynomials of Types II and IV of the previous section. For polynomials of Types I, III, and V, the test results were similar to those for polynomials of Type II, apparently due to the shared Chebyshev factors. The test results on Type IV polynomials indicate the strength of this algorithm in the case of clustered roots.

The number of iterations required and the error bound are displayed in the tables below.

Table 4.10: Number of Iterations and Error Bounds for Hybrid Algorithm on Type II Polynomials

\begin{tabular}{|c|c|c|c|}
\hline $\mathbf{n}$ & $\mathbf{r}$ & Iterations & Errors \\
\hline 64 & 8 & 10 & $3.69 E-10$ \\
\hline 64 & 12 & 23 & $4.96 E-08$ \\
\hline 64 & 16 & 23 & $4.97 E-03$ \\
\hline 128 & 8 & 10 & $2.28 E-11$ \\
\hline 128 & 12 & 28 & $1.97 E-07$ \\
\hline 128 & 16 & 23 & $8.68 E-02$ \\
\hline 256 & 8 & 28 & $6.56 E-12$ \\
\hline 256 & 12 & 28 & $3.64 E-07$ \\
\hline 256 & 16 & 28 & $3.82 E-04$ \\
\hline 512 & 8 & 15 & $8.05 E-13$ \\
\hline 512 & 12 & 28 & $1.71 E-08$ \\
\hline 512 & 16 & 28 & $2.78 E-05$ \\
\hline 1024 & 8 & 28 & $3.72 E-11$ \\
\hline 1024 & 12 & 28 & $1.09 E-08$ \\
\hline 1024 & 16 & 33 & $2.19 E-05$ \\
\hline
\end{tabular}

Table 4.11: Number of Iterations and Error Bounds for Hybrid Algorithm on Type IV Polynomials

\begin{tabular}{|c|c|c|}
\hline $\mathbf{n}$ & Iterations & Errors \\
\hline 64 & 33 & $7.32 E-05$ \\
\hline 128 & 33 & $6.12 E-06$ \\
\hline 256 & 38 & $1.60 E-05$ \\
\hline 512 & 38 & $1.08 E-04$ \\
\hline 1024 & 38 & $9.19 E-01$ \\
\hline
\end{tabular}




\subsection{Tests for the Modular Square Root Iterations (Algorithm 3.4)}

Table 4.12 displays our test results for Algorithm 3.4 that is, for the iterations $f_{i+1}(x) \equiv \frac{1}{2}\left(f_{i}(x)-\right.$ $\left.f_{i}(x)^{-1}\right) \bmod p(x)$, which computed polynomial inverses modulo $p(x)$ by solving the associated Sylvester linear systems of equations. We applied the tests to polynomials of Types I and II.

Already after a small number of iterations, that is, for small integers $i$, the tests have consistently produced polynomials $f_{i}(x)$ whose roots approximated the complex roots of the polynomial $p(x)$ of (1.1) within the fixed tolerance bound $\epsilon=10^{-5}$. At this stage of our tests we applied the MATLAB function "roots()" in order to avoid actual computation of agcds. Namely, as soon as we observed that the polynomial $p(x)$ shared all its complex roots with the polynomial $f_{i}(x)$, we stopped the iterations.

Table 4.12: Number of Iterations for Algorithm 3.4 on Polynomials of Types I and II

\begin{tabular}{|c|c|c|c|}
\hline $\mathrm{n}$ & $\mathrm{r}$ & Type I & Type II \\
\hline 64 & 8 & 9 & 14 \\
\hline 64 & 12 & 4 & 16 \\
\hline 64 & 16 & 2 & 17 \\
\hline 128 & 8 & 9 & 14 \\
\hline 128 & 12 & 12 & 16 \\
\hline 128 & 16 & 2 & 17 \\
\hline 256 & 8 & 9 & 14 \\
\hline 256 & 12 & 12 & 16 \\
\hline 256 & 16 & 8 & 17 \\
\hline 512 & 8 & 9 & 14 \\
\hline 512 & 12 & 12 & 16 \\
\hline 512 & 16 & 8 & 17 \\
\hline 1024 & 8 & 10 & 14 \\
\hline 1024 & 12 & 12 & 16 \\
\hline 1024 & 16 & 11 & 17 \\
\hline
\end{tabular}

Acknowledgements: This work has been supported by NSF Grant CCF-1116736 and PSC CUNY Award 67699-00 45. We are also grateful to Dario A. Bini and two anonymous reviewers, for thoughtful and helpful comments and to Ioannis Z. Emiris and Bernard Mourrain for pointing us out the bibliography on the distribution of real roots of a polynomial.

\section{References}

[1] Ben-Or, M., Tiwari, P.: Simple algorithms for approximating all roots of a polynomial with real roots. J. Complexity, 6(4), 417-442 (1990)

[2] Bini, D.: Numerical computation of polynomial zeros by means of Aberth's method. Numerical Algorithms, 13, 179-200 (1996)

[3] Bini, D. A.: private communication (2014)

[4] Bini, D.A., Boito, P.: A fast algorithm for approximate polynomial GCD based on structured matrix computations. In: Operator Theory: Advances and Applications, vol. 199, pp. 155-173. Birkhäuser Verlag, Basel (2010)

[5] Bini, D. A., Fiorentino, G.: Design, analysis, and implementation of a multiprecision polynomial rootfinder. Numerical Algorithms, 23, 127-173 (2000) 
[6] Bini, D., Pan, V. Y.: Polynomial and Matrix Computations, Volume 1: Fundamental Algorithms. Birkhäuser, Boston (1994)

[7] Bini, D., Pan, V.Y.: Graeffe's, Chebyshev, and Cardinal's processes for splitting a polynomial into factors. J. Complexity, 12, 492-511 (1996)

[8] Bini, D., Pan, V.Y.: Computing matrix eigenvalues and polynomial zeros where the output is real. SIAM J. on Computing 27(4), 1099-1115 (1998) Proc. version in SODA'1991, pp. 384-393, ACM Press, New York, and SIAM Publications, Philadelphia (1991)

[9] Brunie, C., Picart, P.: A fast version of the Schur-Cohn algorithm. Journal of Complexity, $16,1,54-69(2000)$

[10] Bini, D.A., Robol, L.: Solving secular and polynomial equations: a multiprecision algorithm. J. Computational and Applied Mathematics, 272, 276-292 (2014)

[11] Boito, P., Ruatta, O.: Extended companion matrix for approximate GCD. Proc. SNC 2011, pp. $74-80$ (2011)

[12] Cardinal, J.P.: On two iterative methods for approximating the roots of a polynomial. Lectures in Applied Mathematics, vol. 32, pp. 165-188, AMS (1996)

[13] R. M. Corless, S. M. Watt, L. Zhi, QR factoring to compute the GCD of univariate approximate polynomials. IEEE Transactions on Signal Processing, 52, pp. 3394-340 (2004)

[14] C. D'Andrea, A. Galligo, M. Sombra, Quantitative equidistribution for the solutions of systems of sparse polynomial equations, arXiv:1203.1843v3 [math.CV] 6 Aug 2014.

[15] Du, Q., Jin, M., Li, T.Y., Zeng, Z.: The quasi-Laguerre iteration. Math. Computation, 66(217), 345-361 (1997)

[16] Eidelman, Y., Gohberg, I., Haimovici, I.: Separable Type Representations of Matrices and Fast Algorithms, Volumes 1 and 2. Birkhäuser (2013)

[17] Emiris, I. Z., Galligo, A., Tsigaridas, E. P.: Random polynomials and expected complexity of bisection methods for real solving. arXiv:1005.2001 2 [cs.SC] 31 May 2010. Proceedings version in Proceedings of the 2010 International Symposium on Symbolic and Algebraic Computation (ISSAC 2010), 235-242, ACM Press, New York (2010)

[18] Edelman, A., Kostlan, E.: How many zeros of a random polynomial are real? Bulletin AMS, 32(1), 1-37 (1995)

[19] Emiris, I. Z., Pan, V. Y., Tsigaridas, E.: Algebraic algorithms. Chapter 10 of Computing Handbook (Third edition), Volume I: Computer Science and Software Engineering (Allen B. Tucker, Teo Gonzales, and Jorge L. Diaz-Herrera, editors). Taylor and Francis Group (2014). Available at arXiv 1311.3731 [cs.DS]

[20] P. Erd ös and P. Turán, On the distribution of roots of polynomials, Ann. of Math. (2) 51 (1950), 105-119.

[21] Fortune, S.: An iterated eigenvalue algorithm for approximating roots of univariate polynomials. J. of Symbolic Computation, 33(5), 627-646 (2002)

[G90] W. Gautschi, How Unstable are Vandermonde Systems? International Symposium on Asymptotic and Computational Analysis: Conference in Honor Frank W. J. Olver's 65th Birthday (R. Wong, editor), Lecture Notes in Pure and Applied Mathematics, 124, 193210, Marcel Dekker, New York, 1990.

[GI88] W. Gautschi, G. Inglese, Lower Bounds for the Condition Number of Vandermonde Matrices, Numerische Mathematik, 52, 241-250, 1988. 
[22] Golub, G.H., Van Loan, C.F.: Matrix Computations, 4th edition. The Johns Hopkins University Press, Baltimore, Maryland (2013)

[23] Halko, N., Martinsson, P.G., Tropp, J.A.: Finding structure with randomness: probabilistic algorithms for constructing approximate matrix decompositions. SIAM Review 53(2), $217-$ $288(2011)$

[24] Higham, N.J.: Functions of Matrices. SIAM, Philadelphia (2008)

[25] Householder, A.S.: Dandelin, Lobachevskii, or Graeffe. Amer. Math. Monthly, 66, 464-466 (1959)

[26] P. Henrici, Applied and computational complex analysis, Volume 1: Power series?integration?conformal mapping?location of zeros, Wiley (1974).

[27] C. P. Hughes and A. Nikeghbali, The zeros of random polynomials cluster uniformly near the unit circle, Compos. Math. 144 (2008), 734-746.

[28] Kac, M.: On the average number of real roots of a random algebraic equation. Bulletin AMS, 49, 314-320 and 938 (1943)

[29] E. Kaltofen, Z. Yang, L. Zhi, Structured low rank approximation of a Sylvester matrix. Proc. 2nd Intern. Workshop on Symbolic-Numerical Algorithms (SNC 05), 188-201 (Dongming Wang and Lihong Zhi, editors), July 2005, Xi'an, China (2005)

[30] Malajovich, G.,. Zubelli, J. P.: Tangent Graeffe iteration. Numerische Mathematik, 89 (4), 749-782 (2001)

[31] McNamee, J.M.: Numerical Methods for Roots of Polynomials, Part 1 (XIX + 354 pages). Elsevier (2007)

[32] McNamee, J.M., Pan, V.Y.: Numerical Methods for Roots of Polynomials, Part 2 (XXII + 718 pages). Elsevier (2013)

[33] Pan, V.Y.: Complexity of computations with matrices and polynomials. SIAM Review 34(2), 225-262 (1992)

[34] Pan, V.Y.: Optimal (up to polylogarithmic factors) sequential and parallel algorithms for approximating complex polynomial zeros. In: Proc. 27th Ann. ACM Symp. on Theory of Computing, pp. 741-750. ACM Press, New York (1995)

[35] Pan, V.Y.: Approximating complex polynomial zeros: modified quadtree (Weyl's) construction and improved Newton's iteration. J. of Complexity, 16(1), 213-264 (2000)

[36] Pan, V.Y.: Structured Matrices and Polynomials: Unified Superfast Algorithms. Birkhäuser, Boston, and Springer, New York (2001)

[37] Pan, V.Y.: A new proximity test for polynomial zeros. Computers and Math. (with Applications), 41(12), 1559-1560 (2001)

[38] Pan, V.Y.: Numerical computation of a polynomial GCD and extensions. Information and Computation, 167, 2, 71-85 (2001).

[39] Pan, V.Y.: Univariate polynomials: nearly optimal algorithms for factorization and rootfinding. J. Symb. Computations 33(5), 701-733 (2002). Proc. version in ISSAC'2001, pp. 253-267, ACM Press, New York (2001)

[40] Pan, V.Y.: Amended DSeSC power method for polynomial root-finding. Computers and Math. (with Applications), 49 (9-10), 1515-1524 (2005) 
[41] Pan, V.Y.: Univariate polynomial root-finding by arming with constraints. Proc. of the Forth International Workshop on Symbolic-Numerical Computations (SNC '11), San Jose, California, June 2011 (edited by M. Moreno Maza), 112-121, ACM Press, New York (2011)

[42] Pan, V.Y.: Root-refining for a polynomial equation. Proceedings of Workshop on Computer Algebra in Scientific Computing (CASC 2012), (V. P. Gerdt et al. editors), Lecture Notes in Computer Science, vol. 7442, 271-282, Springer, Heidelberg (2012)

[43] Pan, V.Y.: Transformations of matrix structures work again. Linear Algebra and Its Applications, 465, 1-32 (2015)

[44] Pan, V.Y.: Numerical root-finding for a univariate polynomial by means of matrix squaring, preprint (2015)

[45] Pan, V.Y., Qian, G., Yan, X.: Random multipliers numerically stabilize Gaussian and block Gaussian elimination: proofs and an extension to low-rank approximation. Linear Algebra and Its Applications, 481, 202-234 (2015)

[46] Pan, V.Y., Qian, G., Zheng, A.: Real and complex polynomial root-finding via eigensolving and randomization. Proceedings of Workshop on Computer Algebra in Scientific Computing (CASC 2012), (V. P. Gerdt et al. editors), Lecture Notes in Computer Science, vol. 7442, 283-293. Springer, Heidelberg (2012)

[47] Pan, V.Y., Tsigaridas, E.P.: Nearly optimal refinement of real roots of a univariate polynomial. J. of Symbolic Computation, in press. Proc. version in: Proc. Intern. Symposium on Symbolic and Algebraic Computation (ISSAC 2013), (M. Kauers editor), pp. 299-306, Boston, MA, June 2013. ACM Press, New York (2013)

[48] Pan, V.Y., Tsigaridas, E.P.: Accelerated approximation of the complex roots of a univariate polynomial. Proc. of the International Conference on Symbolic Numeric Computation (SNC'2014), 132-134, ACM Press, New York (2014). Also April 18, 2014, arXiv : 1404.4775, revised in August 2015 [math.NA]

[49] Pan, V.Y., Zheng, A.: Root-finding by expansion with independent constraints. Computers and Math. (with Applications), 62, 3164-3182 (2011)

[50] Pan, V. Y., Zhao, L.: Numerically Safe Gaussian Elimination with No Pivoting, Linear Algebra Appl. (2017), http://dx.doi.org/10.1016/j.laa.2017.04.007, arXiv: 1501.05385 [cs.CS], 2 Apr 2017.

[51] Renegar, J.: On the worst-case arithmetic complexity of approximating zeros of polynomials, J. of Complexity 3(2), 90-113 (1987)

[52] Schönhage, A.: The fundamental theorem of algebra in terms of computational complexity. Mathematisches Institut der Universität Tübingen, Germany (1982)

[53] Stewart, G. W.: Matrix Algorithms, Vol II: Eigensystems. SIAM, Philadelphia (2001)

[54] Sagraloff, M., Mehlhorn, K.: Computing real roots of real polynomials. J. of Symbolic Computation, to appear

[55] Schost, E., Spaenlehauer, P.-J.: A quadratically convergent algorithm for structured lowrank approximation. Foundations of Computational Mathematics, to appear

[56] Tilli, P.: Convergence conditions of some methods for the simultaneous computations of polynomial zeros. Calcolo, 35, 3-15 (1998)

[57] Van der Sluis, A.: Upper bounds on the roots of polynomials. Numerische Math., 15, 250-262 (1970) 
[T94] E. E. Tyrtyshnikov, How Bad Are Hankel Matrices? Numerische Mathematik, 67, 2, 261269, 1994.

[58] Vandebril, R., Van Barel, M., Mastronardi, N.: Matrix Computations and Semiseparable Matrices, Volumes 1 and 2. The Johns Hopkins University Press, Baltimore, Maryland (2007 and 2008)

[59] Weierstrass, K.: Neuer Beweis des Fundamentalsatzes der Algebra. Mathematische Werker, Tome III, Mayer und Müller, Berlin, 251-269 (1903)

[60] Watkins, D.S.: The Matrix Eigenvalue Problem: GR and Krylov Subspace Methods. SIAM, Philadelphia, PA (2007)

[61] Winkler, J. R. and Hasan, M.: A non-linear structure preserving matrix method for the low rank approximation of the Sylvester resultant matrix. Journal of Computational and Applied Mathematics, 234, 3226-3242 (2010)

[62] Xi, Y., Xia, J., Cauley, S., Balakrishnan, V.: Superfast and stable structured solvers for Toeplitz least squares via randomized sampling. SIAM J. on Matrix Analysis and Applications, 35, 44-72 (2014)

[63] Xia, J., Xi, J., Gu, M.: A superfast structured solver for Toeplitz linear systems via randomized sampling. SIAM J. on Matrix Analysis and Applications, 33, 837-858 (2012)

\section{Appendix}

\section{A Some Maps of the Variables and the Roots}

Some basic maps of the roots of a polynomial can be computed at a linear or nearly linear arithmetic cost.

Theorem A.1. (Root Inversion, Shift and Scaling, cf. [36].)

(i) Given a polynomial $p(x)$ of (1.1) and two scalars $a$ and $b$, one can compute the coefficients of the polynomial $q(x)=p(a x+b)$ by using $O(n \log (n))$ arithmetic operations. This bound decreases to $2 n-1$ multiplications if $b=0$.

(ii) Reversing a polynomial inverts all its roots by involving no flops, that is,

$$
p_{\text {rev }}(x)=x^{n} p(1 / x)=\sum_{i=0}^{n} p_{i} x^{n-i}=p_{n} \prod_{j=1}^{n}\left(1-x x_{j}\right) .
$$

Note that by shifting and scaling the variable, we can move all roots of $p(x)$ into a fixed disc, e.g., $D(0,1)=\{x:|x| \leq 1\}$.

Theorem A.2. (Dandelin's Root Squaring, cf. [25].)

(i) Let a polynomial $p(x)$ of (1.1) be monic. Then $q(x)=(-1)^{n} p(\sqrt{x}) p(-\sqrt{x})=\prod_{j=1}^{n}\left(x-x_{j}^{2}\right)$.

(ii) One can evaluate $p(x)$ at the $k$-th roots of unity, for $k>2 n$, and then interpolate to $q(x)$ by using $O(k \log (k))$ arithmetic operations overall.

Remark A.1. Recursive root-squaring is prone to numerical stability problems because the coefficients of the iterated polynomials very quickly span many orders of magnitude. It is somewhat surprising, but the Boolean complexity of the recursive root-squaring process is relatively reasonable if high output precision is required [34], [39]. Moreover, one can avoid numerical stability problems and perform all iterations with double precision by applying a special tangential representation of the coefficients and of the intermediate results proposed in [30]. In this case the computations involve more general operations than flops; in terms of the CPU time the computational cost per iteration has the same order as $n^{2}$ flops, performed with double precision. 
Theorem A.3. (The Cayley Maps.)

(i) The map $y=(x-a \sqrt{-1}) /(x+a \sqrt{-1})$, for any real nonzero scalar $a$, sends the real axis $\{x: x$ is real $\}$ onto the unit circle $C(0,1)=\{y:|y|=1\}$.

(ii) The converse map $x=a \sqrt{-1}(1-y) /(y+1)$ sends the unit circle $C(0,1)$ onto the real axis.

\section{B Some Functional Iterations for Polynomial Root-finding}

Newton's and Ehrlich-Aberth's are two celebrated functional iteration processes for the approximation of a single root of a polynomial $p(x)$ of (1.1) and all its roots, respectively. They are highly efficient and popular, but not specialized to our task of approximating real roots, and we only use them as auxiliary root-refiners.

Hereafter a disc $D(X, r)$ is said to be $\gamma$-isolated for a polynomial $p(x)$ and $\gamma>1$ if it contains all roots of the polynomial lying in the disc $D(X, \gamma r)$. In this case we say that the disc has isolation ratio at least $\gamma$.

Newton's iterations refine an approximation $y^{(0)}$ to a single root of a polynomial $p(x)$ of (1.1),

$$
y_{0}=c, y^{(h+1)}=y^{(h)}-p\left(y^{(h)}\right) / p^{\prime}\left(y^{(h)}\right), h=0,1, \ldots
$$

Ehrlich-Aberth's iterations refine $n$ simultaneous approximations $z_{1}^{(0)}, \ldots, z_{1}^{(n)}$ to all $n$ roots $x_{1}, \ldots, x_{n}$ of such a polynomial,

$$
z_{i}^{(h+1)}=z_{i}^{(h)}-1 / e_{i}^{(h)}, \text { for } e_{i}^{(h)}=p\left(z_{i}^{(h)}\right) / p\left(z_{i}^{(h)}\right)-\sum_{j \neq i} \frac{1}{z_{i}^{(h)}-z_{j}^{(h)}}, i=1, \ldots, n,
$$

See [31, 32 for various other functional iterations.

As we can see next, both iterative algorithms refine very fast the crude initial approximations to simple isolated roots of a polynomial.

Theorem B.1. Assume a polynomial $p=p(x)$ of (1.1) and let $0<3(n-1)\left|y_{0}-x_{1}\right|<\left|y_{0}-x_{j}\right|$, for $j=2, \ldots, n$. Then Newton's iterations (B.1) converge to the root $x_{1}$ quadratically right from the start, namely, $\left|y_{k}-x_{1}\right| \leq 2\left|y_{0}-x_{1}\right| / 2^{2^{k}}$, for $k=0,1, \ldots$.

Proof. See [56, Theorem 2.4], which strengthens [51, Corollary 4.5].

Theorem B.2. (See [56, Theorem 3.3].) Assume a polynomial $p=p(x)$ of (1.1) and crude initial approximations $y_{j}^{(0)}$ to the roots $x_{j}$ such that $0<3 \sqrt{n-1}\left|y_{j}^{(0)}-x_{j}\right|<\left|y_{j}^{(0)}-y_{i}^{(0)}\right|$, for $i \neq j$, $j=1, \ldots, n$. Then Ehrlich-Aberth's iterations converge to the roots $x_{j}$ with the cubic rate right from the start, namely, $\left|y_{j}^{(k)}-x_{j}\right| \leq\left|y_{j}^{(0)}-x_{j}\right| /\left(2^{3^{k}} \sqrt{(n-1)}\right)$, for $j=1, \ldots, n$ and $k=0,1, \ldots$

The paper [56] also proves quadratic convergence of the WDK iterations to all $n$ roots, lying in some given discs with an isolation ratios at least $3(n-1) / 8$. These iterations are due to Weierstrass 59, but are frequently attributed to its later re-discoveries by Durand in 1960 and Kerner in 1966.

By exploiting the correlations between the coefficients of a polynomial and the power sums of its roots, the paper 48 had weakened the above assumptions on the initial isolation. More precisely, assuming that a simple root lies in the disc $D(0,1)$ and that the disc has an isolation ratio at least $s \geq 1+1 / \log _{2}(n)$, the paper 48 increases it to $c n^{d}$, for any fixed pair of constants $c$ and $d$, at the arithmetic cost $O(n)$, and similarly increased the isolation ratio of the $n$ discs covering all the $n$ roots at the arithmetic cost $O\left(n \log ^{2}(n)\right)$.

In the case of a single disc, one can assume even an isolation ratio $s \geq 1+c^{\prime} / n^{d^{\prime}}$, for any pair of constants $c^{\prime}$ and $d^{\prime}$, and then increase it to $s \geq c n^{d}$, for any other pair of constants $c$ and $d$, at the arithmetic cost $O\left(n \log ^{2}(n)\right)$. Indeed one can achieve this by performing $h$ root-squaring iterations of TheoremA.2 for $h$ of order $\log (n)$ because each squaring of the roots also squares the isolation ratio. This lifting process ensures the desired isolation for the lifted roots of the new lifted polynomial, but the descending back to the original roots can be also achieved by using $O\left(n \log ^{2}(n)\right)$ arithmetic 
operations [34, 39. We refer the reader to Remark A.1 on the precision growth in these iterations and their Boolean complexity.

Can we completely relax the assumption of the initial isolation? Empirically fast global convergence (that is, convergence right from the start) is very strong over all inputs for the WDK, Ehrlich-Aberth, and some other iterations that approximate simultaneously all $n$ roots of a polynomial $p(x)$ of (1.1). The papers [41, [49, and [42] have challenged the researchers to support this observation with a formal proof, which is still missing, however.

\section{Fast Root-finding Where All Roots Are Real}

Theorem C.1. Assume that all roots of a polynomial $p(x)$ of (1.1) are real.

(i) Then the modified Laguerre algorithm of [15] converges to all of them right from the start, uses $O(n)$ flops per iteration, and therefore approximates all the $n$ roots within $\epsilon=1 / 2^{b}$ by using $O(\log (b))$ iterations and performing $O(n \log (b))$ flops.

(ii) The latter asymptotic arithmetic cost bound is optimal and is supported by the alternative algorithms of [1] and [8] as well.

(iii) All these algorithms reach the optimal Boolean cost bound up to polylogarithmic factors.

\section{Counting the Roots in a Disc. Root Radii, Distances to the Roots, and the Proximity Tests}

In this subsection we estimate the distances to the roots of $p(x)$ from a complex point and the number of the roots in an isolated disc.

The latter task can be solved by using the following result from [51, Lemma 7.1] (cf. also [26], [52, Theorem 14.1] and [9]).

Theorem D.1. [51, Lemma 7.1] It is sufficient to perform FFT at $n^{\prime}=16\left\lceil\log _{2} n\right\rceil$ points (using $1.5 n^{\prime} \log \left(n^{\prime}\right)$ flops) and $O(n)$ additional flops and comparisons of real numbers with 0 in order to compute the number of roots of a polynomial $p(x)$ of (1.1) in a 9-isolated disc $D(0, r)$.

Remark D.1. The algorithm of [51] supporting Theorem D.1 only uses the signs of the real and imaginary parts of the $n$ output values of FFT. For some groups of the values, the pairs of the signs stay invariant and can be represented by a single pair of signs. Can this observation be exploited in order to decrease the computational cost of performing the algorithm?

Corollary D.1. It is sufficient to perform $O(h n \log (n))$ flops and $O(n)$ comparisons of real numbers with 0 in order to compute the number of roots of a polynomial $p(x)$ of (1.1) in an s-isolated disc $D(0,1)$, for $s=9^{1 / 2^{h}}$ and any positive integer $h$.

Proof. Every root-squaring of Theorem A.2 squares all root-radii and the isolation ratios of all discs $D(0, r)$, for all positive $r$. Suppose $h$ repeated squaring iterations map a polynomial $p(x)$ into $p_{h}(x)$, for which the disc $D(0,1)$ is 9 -isolated. Then, by applying Theorem D.1 we can compute the number of roots of $p_{h}(x)$ in this disc, equal to the number of roots of $p(x)$.

In view of Remark A.1. one must apply the slower operations of 30 or high precision computations in order to support even a moderately long sequence of root-squaring iterations, but in some cases it is sufficient to apply Corollary D.1, for small positive integers $h$. Note that $9^{1 / 2^{h}}$ is equal to $1.3160 \ldots$, for $h=2$, to $1.1472 \ldots$, for $h=3$, to $1.0710 \ldots$, for $h=4$, and to $1.0349 \ldots$, for $h=5$.

We can use the following result if we agree to perform computations with extended precision.

Theorem D.2. (The Root Radii Approximation.)

Assume a polynomial $p(x)$ of (1.1) and two real scalars $c>0$ and $d$. Define the $n$ root radii $r_{j}=\left|x_{k_{j}}\right|$, for $j=1, \ldots, n$, distinct $k_{1}, \ldots, k_{n}$, and $r_{1} \geq r_{2} \geq \cdots \geq r_{n}$. Then, by using $O\left(n \log ^{2}(n)\right)$ arithmetic operations, one can compute $n$ approximations $\tilde{r}_{j}$ to the root radii $r_{j}$ such that $\tilde{r}_{j} \leq r_{j} \leq$ $\left(1+c / n^{d}\right) \tilde{r}_{j}$, for $j=1, \ldots, n$. 
Proof. (Cf. 52, [35, Section 4].) At first fix a sufficiently large integer $k$ and apply $k$ times the root-squaring of Theorem A.2, which involves $O(k n \log (n))$ arithmetic operations. Then apply the algorithm of [52] (which uses $O(n)$ arithmetic operations) in order to approximate within a factor of $2 n$ all root radii $r_{j}^{(k)}=r_{j}^{2^{k}}, j=1, \ldots, n$, of the output polynomial $p_{k}(x)$. By taking the $2^{k}$-th roots, approximate the root radii $r_{1}, \ldots, r_{n}$ within a factor of $(2 n)^{1 / 2^{k}}$, which is $1+c / n^{d}$, for $k$ of order $\log (n)$.

Alternatively we can approximate the root radii by applying the semi-heuristic method of [2, used in the packages MPSolve 2000 and 2012 (cf. [5] and [10) or by recursively applying Theorem D.1 although neither of these techniques support competitive complexity estimates.

The following two theorems define the largest root radius $r_{1}$ of the polynomial $p(x)$.

Theorem D.3. (See [57].) Assume a polynomial $p(x)$ of [1.1). Write $r_{1}=\max _{j=1}^{n}\left|x_{j}\right|, r_{n}=$ $\min _{j=1}^{n}\left|x_{j}\right|$, and $\gamma^{+}=\max _{i=1}^{n}\left|p_{n-i} / p_{n}\right|$. Then $\gamma^{+} / n \leq r_{1} \leq 2 \gamma^{+}$.

Theorem D.4. (See [37].) For $\epsilon=1 / 2^{b}>0$, one only needs $a(n, \epsilon)=O(n+b \log (b)$ ) flops to compute an approximation $r_{1, \epsilon}$ to the largest root radius $r_{1}$ of $p(x)$ such that $r_{1, \epsilon} \leq r_{1} \leq 5(1+\epsilon) r_{1, \epsilon}$. In particular, $a(n, \epsilon)=O(n)$, for $b=O(n / \log (n))$, and $a(n, \epsilon)=O(n \log (n))$, for $b=O(n)$.

Both theorems can be immediately extended to the approximation of the smallest root radius $r_{n}$ because it is the reciprocal of the largest root radius of the reverse polynomial $p_{\text {rev }}(x)=x^{n} p(1 / x)$ (cf. Theorem A.1). Moreover, by shifting a complex point $c$ into the origin (cf. Theorem A.1), we can turn our estimates for the root radii into the estimates for the distances to the roots from the point $c$. Approximation of the smallest distance from a complex point $c$ to a root of $p(x)$ is called the proximity test at the point. One can perform such a test by applying Theorems D.1, D.3, or D.4

Alternatively, for heuristic proximity tests by action at a point $c$ or at $n$ points, one can apply Newton's iterations (B.1) or an appropriate functional iterations, such as the Ehrlich-Aberth iterations (B.2), and estimate the distance to the roots by observing convergence or divergence of the iterations.

Theorem D.4 and all these iterations, including Newton's, Ehrlich-Aberth's and WDK's, can be applied even where a polynomial $p(x)$ is defined by a black box subroutine for its evaluation rather than by its coefficients. 